\title{
Computation and the Ambiguity of Perception
}

\author{
Jonathan Cohen
}

Sometimes towers which had looked round from a distance appeared square from close up; and enormous statues standing on their pediments did not seem large when observed from the ground.

— Descartes, Meditation VI, CSM:II, 53.

There is a class of phenomena that suggests strongly that perception is, in some sense to be explained, ambiguous in what it tells us about the world. In the cases at issue, the perceptual system is capable of responding to a single stimulus - say, as manifested in the ways in which subjects sort that stimulus - in different ways. Indeed, in many cases, subjects can be made to switch at will between these different modes of response. This paper is about that ambiguity, and about how it should be characterized and accounted for within a general theory of perception.

\section{The Puzzle Cases: The Ambiguity of Perception}

Let me begin, then, by bringing to mind some (I hope) familiar cases in which our perceptual systems generate multiple reactions to one stimulus. ${ }^{1}$ (As usual in such discussions, I'll be focusing below mostly on visual perception; but I suspect that much of what I say can be generalized to at least some other perceptual modalities as well.)

A first classic example is that of the non-uniformly illuminated but uniformly painted wall (e.g., as in figure 1). Consider two contiguous and samesized, same-shaped regions of the wall in figure 1 that are each (roughly) illuminated uniformly, but such that there is a difference in the illumination between the two - one is in shadow and one is in direct sunlight. Here is a fact. Subjects perceiving this stimulus condition can indicate about the differently illuminated regions (say, by their sorting behavior) that they are, in one way, alike in their color appearance and that they are, in another way, not alike in their color appearance. It seems that visual systems can pick up both the constancy/similarity or the inconstancy/dissimilarity between the regions, and subjects can respond (say, in the ways that they sort, report, or make matches) to either one. Moreover, famously, ordinary subjects can 
be made to switch between these different modes of response simply by manipulating the task instructions (Arend and Reeves, 1986; Blackwell and Buchsbaum, 1988; Valberg and Lange-Malecki, 1990; Arend et al., 1991; Troost and deWeert, 1991; Cornelissen and Brenner, 1995; Bäuml, 1999). These familiar results give us powerful reason for believing that, in the kind of case at issue, perception is representing distinct things about the single distal stimulus. Assuming (standardly, though not uncontroversially) that perceptual states have representational contents, we can put this point by saying that the perceptual states caused by such stimuli have (at least) two different contents - one representing the aspect of similarity driving one kind of subject response, and one representing the aspect of dissimilarity driving the other kind of subject response. ${ }^{2}$

[Put figure 1 about here.]

Here is another familiar example of the same sort of phenomenon. Viewing two telephone poles at different distances, subjects can judge of the different poles both that they are, in one way, alike in their size appearance and that they are, in another way, not alike in their size appearance (see figure 2). And, again, ordinary subjects can be made to report on (or respond in a quantitatively measurable way that is sensitive to) either the constancy/similarity or the inconstancy/dissimilarity simply by manipulating the task instructions (for a review of relevant literature, see Wagner, 2006, chapter 6). Here again, it is natural to regard subjects' distinct judgments about the single stimulus as reflecting different contents that perception represents. On the one hand, perception represents the aspect of similarity driving one kind of subject response; and, on the other hand, perception represents the aspect of dissimilarity driving the other kind of subject response.

[Put figure 2 about here.]

A final, and equally familiar, example of the phenomenon involves the visual perception of penny held at two different angles: one head on (i.e., so that one's line of sight is perpendicular to the surface of the penny), and one tilted (i.e., so that one's line of sight forms an acute angle with the surface of the penny), as in figure 3. ${ }^{3}$ Yet again, we find that ordinary subjects can be made to report on (or respond in a quantitatively measurable way that is sensitive to) either a respect in which the apparent shape of the plate is constant/similar between the two presentations, or a respect in which the apparent shape of the plate is inconstant/dissimilar between the two presentations. And yet again, this gives us reason to believe that visual perception can represent both an aspect of similarity and an aspect of dissimilarity between the two presentations of the plate.

[Put figure 3 about here.]

It seems, then, that in these familiar cases (and many others that I won't bother to describe), subjects can behave in quite different ways as a result of perceiving one stimulus. They have a reaction that recognizes the simultaneously perceived wall regions/telephone poles/dinner plates as alike as constant - in the relevant aspect of their appearance. And they have a reaction that recognizes the simultaneously perceived wall regions/telephone 
poles/dinner plates as unlike — as inconstant - in the relevant aspect of their appearance. We can call the former the constancy reaction, and the latter the inconstancy reaction. These two reactions are not simply different from one another. They are contrary, or opposed, to one another. Therefore, the fact that both are simultaneously present in a single perceptual system upon perceiving a single stimulus is quite puzzling. Thus, I'll label cases in which both of these sorts of reactions are available as puzzle cases. My central question in this paper will be how we should think about these puzzle cases. ${ }^{4}$

While there is much that is unclear about the perception of puzzle cases, one feature I want to emphasize immediately is that the perceptual system does not respond to these cases by representing a contradiction. This can be seen by comparison with, for example, the perceptual representation of the spatial layouts in certain familiar Escher drawings containing inconsistent cues about the depth relationships obtaining between depicted objects. Similarly, some have treated the waterfall illusion as another case where the perceptual system attributes and withholds a single property (viz., the property of moving with respect to other objects in the scene) to an object, resulting in a contradictory perceptual content. The right thing to say about the perception of such Escher drawings, and perhaps about the waterfall illusion as well, is that the stimulus causes the perceptual system to attribute conflicting properties (or to attribute and withhold a single property); and when this result obtains, there is an interesting and recognizable (if poorly understood) perceptual breakdown. The point I am stressing here is that the perceptual phenomena involving our puzzle cases contrast with our perceptual reactions in the Escher cases: the former do not seem to involve the perceptual representation of contradiction that we undergo in the latter.

The contrast between the puzzle cases and perceptual contradiction makes for a further constraint on our treatment of the former. For it is not so obvious how we can account for the dual contents that seem to be present in the puzzle cases without treating them as cases of perceptual contradiction. It is curious that, for example, subjects can both sort the shaded and the unshaded regions of the unevenly illuminated wall as similar in color and as dissimilar in color, given the usual thought that two objects cannot be of the same color and of different color. Or, again, it is curious that subjects can both sort the two telephone poles as similar in size and sort the same telephone poles as dissimilar in size, given that two objects cannot be of the same size and different size. And it is curious that subjects can both sort the tilted dinner plate with circles seen head on and sort the same tilted dinner plate with ellipses seen head on, given that being circular and being elliptical are incompatible determinates of a common determinable. But it is yet more curious that they can do these things without representing a contradiction!

What should we make of this situation? 


\section{A Computational Account}

I want to begin with the idea that the distinct behaviors exhibited in our puzzle cases arise because perceptual systems represent concrete physical individuals (walls, telephone poles, pennies) exemplifying multiple properties. This acceptance of multiple (dual) property representations by perception (some version of which is accepted by Tye $(1996,2002)$; Byrne (2002); Noë (2004); Schellenberg (2008), among others) has a lot to recommend it. ${ }^{5}$ For one thing, because it explicitly recognizes distinct contents, the dual property view contains resources that allow it to account for the bimodal behavior of subjects in our puzzle cases, and in this way is superior to views recognizing only a single content, which are consequently unable to account for the full range of observed behavior in such cases. For another, in treating the cases as involving the attribution of multiple properties to one and the same external, ordinary individual, dual property views also avoid the ontological costs associated with the introduction of internal, exotic entities such as sensedata. Moreover, such views avoid the well-known difficulties of adverbialist accounts according to which there are no individual objects of perception at all (Ducasse, 1942; Chisholm, 1957; Sellars, 1963, 1975).

Despite these relative advantages, there remains the outstanding question of how a dual representation view of the sort I have in mind can avoid treating the puzzle cases at issue as involving perceptual contradiction. While proponents of the dual representation view have not always faced up to this problem explicitly, their choice of terminology suggests the strategy of drawing some sort of distinction between the distinct perceptually represented properties e.g., the penny's roundness is sometimes described as an "object" or "distal" property, while its ellipticality is an "apparent," "proximal," "perspectival," or "situation-dependent" property. But of course the mere labels do not amount to a substantive answer to our question so much as an invitation to explain the difference. How, then, should someone sympathetic to the dual representation account explain the distinction between the properties represented?

In what follows I want to propose an answer to this question, and, ultimately, an account of the puzzle cases, in terms of computation. In particular, I'll suggest that we should supplement the appeal to multiple representations with a computational conception of how they are related to one another. I hope that the broad outlines of this computational treatment (if not its specific details) will strike many readers as uncontroversial or even obvious. Still, I believe that there are several reasons for thinking through this computational view. Among other benefits, the computational outlook suggests a natural explanation of the sense in which the target properties are treated differently by the perceptual system. Namely, they are treated differently by being assigned different roles in a certain functional/computational relationship that perceptual systems compute. More specifically, I suggest that one of our properties is the input to a computation carried out by perceptual systems, and the other property is the output of that computation. This, in turn, will allow us to understand the bimodal pattern of subject responses that we have noted 
without wrongly predicting that the puzzle cases will lead to the perceptual representation of contradiction, and without falling prey to the problems that have plagued alternative proposals. Ultimately, I think the computational outlook will put us in a better position to understand just which properties are perceived in the puzzle cases, just which properties are represented by the perceptual system, and how they are related to one another.

\subsection{Perception and Computation}

Let us, then, begin to consider our puzzle from the point of view of the perceptual system and the computations it carries out.

For concreteness, consider the tilted penny once again. Suppose it is correct that perception attributes to the penny one property that comes out in our constancy reaction (call it round) and a different property that comes out in our inconstancy reaction (call it elliptical). ${ }^{6}$ We know these properties can't both be treated as determinates of a common determinable (say, shape) on pain of the kind of reaction to perceptual contradictions that is simply not present in the case. So our question is: how does perception treat the two properties distinctly? And the answer to this question that the computational view provides is: perception treats the two properties as playing different roles in a certain computational relationship.

To see what this amounts to, start by thinking about the gross state of the perceptual system at a time - henceforth, the perceptual state. The perceptual state is obviously a property of the perceptual system, as opposed to distal, worldly items. On the other hand, distal, worldly items that we perceive have dispositions to cause those perceptual states in us under certain circumstances; and these dispositional properties - henceforth, perceptual state dispositions - are properties of distal, worldly items as opposed to perceptual systems.

Consider, in particular, the perceptual state resulting from the subject's visually attending to the tilted penny. The system begins by transducing, and then representing instances of certain properties. This transduction step can be understood as being caused to enter into a certain perceptual state type - namely, the very perceptual state type whose tokens are caused in us by (inter alia) visually attending to ellipses head on. But of course what the system attributes to the worldly, distal penny is not a property of perceptual systems. Rather, it represents (and is ordinarily warranted in representing) the worldly, distal item as having the disposition to cause that type of perceptual state. That is to say, it represents the distal item as bearing this perceptual state disposition: disposed to generate in us an instance of the type of perceptual state we undergo when perceiving an ellipse straight on. That perceptual state disposition, I claim, is the property we were earlier (and, we can now see, misleadingly) labeling as elliptical. Significantly, given the representation of this perceptual state disposition, together with other represented information about the scene, the perceptual system can do something else. Namely, it can carry out a computation whose output is a representation with the content that there is a distal instance of round. Crucially, the two properties disposed to generate in us an 
instance of the type of perceptual state we undergo when perceiving an ellipse straight on and round play entirely different roles in this computation: the former is input, and the latter is output. ${ }^{7}$

Thinking of perception in such computational terms provides a ready explanation of the distinct behaviors of subjects in puzzle cases. The current proposal accounts for the inconstancy reactions of perceivers - the reaction that distinguishes between the tilted penny and the penny seen straight on, and that assimilates the tilted penny to an oval seen straight on - by appeal to representations of perceptual state dispositions. For the tilted penny and the oval seen straight on share a perceptual state disposition that the penny seen straight on lacks (viz., disposed to generate in us an instance of the type of perceptual state we undergo when perceiving an ellipse straight on). On the other hand, we have the resources to account for the constancy reaction that is also present in subjects by appeal to their representation of object features. For the tilted penny and the penny seen straight on share an object feature that the oval seen straight on lacks (viz., round). In this case, the geometric shape of the penny (it is round) is one of the factors that determines the subject's total perceptual state, and so is something that the perceptual system can compute given sufficient information about the perceptual perspective. ${ }^{8}$

Accepting the foregoing also puts us in a position to explain the difference between ordinary puzzle cases and cases involving the perceptual representation of a contradiction. When we perceive the perceptually contradictory Escher drawings, we attribute incompatible properties to one and the same object. When, in contrast, we perceive the tilted penny, we attribute compatible properties. There is no inconsistency in a thing's being (or being represented as being) simultaneously round and disposed to generate in us an instance of the type of perceptual state we undergo when perceiving an ellipse straight on. And, to repeat, the perceptual system can distinguish the two properties - and can associate each with the appropriate determinable - by assigning them different roles in the computational process it carries out.

\subsection{Computation and The Perspectival Character of Perception}

Why do perceptual systems carry out these computations? They do so because such computations are inevitable for the recovery of object features (which is, as it happens, one of the important roles that perception fills) given the perspectival perceptual relation to the world that we happen to enjoy.

In saying that our perceptual relation to the world is perspectival, I mean, among other things, that we always perceive objects and their properties from a particular angle and distance, under particular lighting conditions, in the context of a particular cognitive and perceptual history and future that affects the particular state of adaptation of our transducers, our perceptual expectations, and on and on. That perception is perspectival in these, and other, ways is a permanent, mandatory fact of our condition. While it may be that there is a species of the kind perception - perhaps a species available to God - that is not perspectival in this sense, it seems to be true and 
necessary that perception in creatures like us (as we in fact are) always occurs under a perspective. Moreover, it turns out that a difference in perspective is (very often) a difference that makes a discriminable difference to perceptual systems. ${ }^{9}$

For this reason, the perceptual state is an interaction effect resulting from the (typically independent) contributions of two different classes of features: object features, which qualify perceived objects, and perspectival features which constitute the perceptual circumstances under which we perceive objects. ${ }^{10}$ Although it is difficult to characterize the contrast between perspectival and object features in more precise terms, I take it that the basic idea is familiar enough as a principle of experimental design in perception science. In crude terms, the idea is that our perceptual responses depend systematically on both the way the world is (object features) and how we as perceivers are situated within that world (perspectival features), and that, consequently, any attempt to account for our perceptual responses has to control for these features separately.

That things are so arranged in the world explains why perceptual systems do the computational things that I am claiming they do. Since the perceptual state is a joint product of perspectival features and object features, computation on the basis of the perceptual state (the input) is needed to recover representations of object features or perspectival features (about which see more below) as output. Luckily, the perceptual state will be fixed by causal interaction with the world. Consequently, the perceptual state will be available for use as a computational input (as will the dispositional externalization of that state, the perceptual state disposition). Hence the perceptual system is in a position to carry out the needed computations: given either perspectival or object features, the system can compute the other. ${ }^{11} 12$

\subsection{What Is Represented?}

In the foregoing I have focused mainly on two perceptual reactions: the constancy reaction (which I explained in terms of the stability of object features) and the inconstancy reaction (which I explained in terms of the instability of perceptual states/perceptual state dispositions). The behavioral evidence, then, gives us reason for believing that the two sorts of variables enlisted to explain the behavior (viz., object features and perceptual state dispositions) are in fact represented by perceptual systems. But the computational framework set out above makes use of not two but three sorts of variables - in addition to variables corresponding to object features and perceptual states, it appeals to variables corresponding to perspectival features. Thus, it would be nice to see some independent empirical confirmation of the existence of representations of the latter. In particular, if it is true that perspectival features are represented by the perceptual system, as I am claiming, it would be nice to see some examples of cases where subjects' reactions tracked those features rather than object features or perceptual state dispositions. Luckily, there appear to be such examples. 
For example, there is strong evidence that subjects represent the perspectival feature of illumination in color matching tasks. For one thing, subjects can, when asked, make matches of perspectival features, such as ambient illumination, as opposed to object features, such as surface lightness (Katz, 1935; Gilchrist, 1988; Hurlbert, 1989; Jameson and Hurvich, 1989; Zaidi, 1998). Indeed, subjects can even characterize the different incident illumination in the two regions of a scene - say, between a swath of forest in a scene illuminated by direct sunlight and the swath of forest in the same scene illuminated by partially could-obscured sunlight (Arend, 1993; Zaidi, 2001, 1998). These facts give us reasonably direct reasons for believing that subjects represent perspectival features at least some of the time.

A second sort of (less direct) evidence for the same conclusion comes from the "tissue-contrast" effect discussed by von Helmholtz $(1962,547)$. Mausfeld (2003) describes the effect this way:

If a small piece of grey paper, to which we can refer as a test spot, is placed on the centre of a large piece of coloured paper and a piece of tissue paper is then placed over these pieces of paper, the test spot has a colour appearance roughly complementary to the colour of the surrounding piece of paper (while an induced colour is absent or much weaker without the tissue paper). Often, as was also noticed by Helmholtz, the complementary colour of the test spot is much more vivid than the weak colour of the surrounding piece of paper... The tissue paper phenomenon behaves as if the chromatic content of the surround is captured by the spatial layer of the tissue and then interpreted as a chromatic illumination (415; cf. Mausfeld (1998)).

Again, it is hard to see why the properties of the surround should influence subjects' representation of the illumination, as they appear to, without supposing that subjects are representing the perspectival feature of illumination.

Another piece of support for the same conclusion is the finding that subjects' achromatic point settings for infields inside chromatic surrounds are systematically skewed toward the chromaticity of the surround (Walraven, 1976; Shevell, 1978); again, this result is hard to interpret without supposing that subjects are representing illumination information (and that the properties of the surround influence this representation).

A final and related result is that, in cases involving the perception of infields in chromatic surrounds, the point in chromaticity space on which constant hue lines converge is not the achromatic point but the chromaticity of the surround (Ekroll et al., 2002). Yet again, this finding suggests that the perceptual system is parsing out a feature of the artificial stimulus (viz., the chromatic content of the surround) as reflecting a perspectival feature (presumably something like illumination, or perhaps a quality of an intervening mist) rather than an object feature of the test spot/infield. Therefore, these cases strongly suggest that perceptual systems do (at least in many cases) represent perspectival features. ${ }^{13}$ 
Thus, there is evidence that perceptual systems can represent object features, perspectival features, and perceptual state dispositions. That is, the evidence shows that perceptual systems can be, in particular situations, responsive to any of the three. But what the evidence marshaled so far does not show is whether all of these sorts of features are in fact represented in every perceptual episode. Of course, the perceptual system always has a gross state at a time; consequently, whenever there is an object of perception, there is a good sense in which the perceptual system, just by being in such a gross state, is representing a perceptual state disposition of its object. But it is not obvious that perceptual systems always represent object features and perspectival features - it is not obvious that they always carry out the computations need to arrive at an explicit representation of object or perspectival features. ${ }^{14}$

Now, one might think that perceptual systems must, at the very least, represent object features whenever they track and reidentify objects in a changing world. The thought would be that object features are a much more reliable clue for tracking and reidentification than perceptual state dispositions, since the latter can be shared by a variety of qualitatively distinct objects perceived under different perceptual conditions. But I think this line of thought is too quick. I believe we have good reasons for thinking that, in certain conditions, perceptual systems can track and reidentify on the basis of perceptual state dispositions and perspectival features without ever representing object features. For example, with respect to color perception, Zaidi (1998, 2001), Foster and Nascimento (1994), and Dannemiller (1993) have suggested that we might track objects perceived at different times not by comparing their object features, but by comparing their perceptual state dispositions in light of what we can estimate about the illumination. Crudely, the idea is that we can ask whether the two perceptual state dispositions lie in the graph of transformations that correspond to ecologically realistic changes in perspectival features (here, illumination). In other words, on these proposals, the perceptual system answers the question about object identity by asking whether perceptual state dispositions vary systematically in a way that could be accounted for by a known change in illumination. It does so on the basis of qualitatively distinct perceptual state dispositions, and without requiring the computation of object features. If something like this proposal is accurate, then we have further reason for denying that the perceptual system must routinely compute object features - even in situations where it must track or reidentify objects over time.

\subsection{Computation and Inference}

Abstracting from the details, what the computational account says is that the perceptual system is engaged in (possibly among other things) moving from the representation of one kind of feature (perceptual state dispositions) to another (object features, perspectival features). Thought of in this way, the computational conception of perception invites comparison with another muchstudied form of computational transition between representational states - 
viz., inference. What gives this question its interest is that, because inference is traditionally conceived as cognitive rather than perceptual, the comparison invites us to wonder whether the computational conception of perception might end up construing much or all of perception in a way that looks excessively conceptual or post-perceptual. Specifically, the thought might be that, contrary to the picture on offer here, it might be more accurate to think of the perceptual system simply as supplying a perceptual state disposition, from which general cognitive (rather than perceptual) inferential mechanisms arrive at a representation of object features or perspectival features.

I want to resist the assimilation of the computational account of perception to a species of inference, at least as the latter is traditionally understood.

To begin to see why, first note that, if the "perceptual" computations we have been discussing are after all inferential, then it seems reasonably clear that they should not be regarded as conscious inferences. For one thing, I take it there is no conscious awareness of such transitions. Moreover, in many ordinary cases where the transitions occur there seems to be no conscious awareness of the perceptual state dispositions that are their input. For that matter, in many ordinary cases where we know the transitions occur because they have systematic effects on ensuing behavior - e.g., in the orientation of grasp, in perceptual priming, etc. - there is no conscious awareness of the object/perspectival features that are their output. Thus, there seems very little ground for treating such computations as conscious inferences.

Could they, however, be treated as unconscious inferences? I doubt it. One consideration that tells against the unconscious inference view is that we see constancy reactions (that depend on the relevant computations) in comparatively cognitively unsophisticated creatures. For example, there appears to be size constancy (at least at relatively short distance ranges) in newborn humans (Granrud, 2006; Slater et al., 1990) in non-human primates (Fujita, 1997; Barbet and Fagot, 2002), goldfish (Douglas et al., 1988), and amphibians (Ingle, 1998). Similarly, color constancy is present in goldfish, honeybees, (and several other non-human animals; see the review in Neumeyer (1998)) and human infants somewhere between 9 and 20 weeks old (Dannemiller and Hanko, 1987), Dannemiller (1989). Since there is evidence of our computations in such creatures, but not corresponding evidence of general (unconscious) cognitive inference in them, this gives us reason for resisting treating the former as species of inference. On the other hand, although the evidence is much more limited, there appear to be cases where the computations we are discussing are impaired but where general cognitive inference and other cognitive capacities are spared (on the selective impairment of certain kinds of size constancy, see Cohen et al. (1994); on the selective impairment of color constancy, see Rüttiger et al. (1999)). Since there appears to be double-dissociation between the capacity to carry out our computations and the capacity for unconscious cognitive inference, it is hard to accept that the former is an instance of the latter.

A second kind of consideration that tells against treating our computations as cognitive inferences is that the former appear to be informationally encapsu- 
lated in a way that inference is not (Fodor, 1983). Thus, it is significant that we persist in mis-computing object sizes from the perceptual state dispositions of objects in the Muller-Lyer illusion, the Ponzo illusion, Ebbinghaus illusion, etc., even after learning about those specific cases and the errors we are prone to in those sorts of situations. But if the computations carried out were unconscious cognitive inferences, then learning about the cases should make available new premises that would prevent us from making the inferences we had made prior to learning about them. That is, since (conscious and unconscious) inference is labile to cognitively represented information, introducing new information (by learning) should modify what is and what is not inferred. But our computations survive the introduction of new (and highly relevant) information that would have defeated them had them had they been inferential. Again, this makes it the inferential view deeply implausible. ${ }^{15}$

\section{Conclusion}

The puzzle cases are interesting and important because they give us a glimpse of (some of) the disparate sorts of things that perception does. In this paper I have argued that the best hope of accounting for the disparate behavior of perception lies in recognizing the perceptual representation of disparate sorts of features and the computational connections that hold between them. Besides allowing for a satisfactory treatment of the puzzle cases, the resulting computational treatment of perception is independently motivated by considerations about the ineluctably perspectival condition in which we find ourselves in the world, and makes (testable) predictions about possibly unexpected representational states. For these reasons, it seems to me that the computational proposal offered here gives us a rich and sophisticated way of thinking about perception. ${ }^{16}$ 


\section{Notes}

${ }^{1}$ I prefer to bring out the ambiguity at issue by pointing to clearly bimodal experimental results in matching tasks rather than by the kinds of raw appeals to introspective phenomenology favored by, e.g., Noë (2007); Kelly (2004). I believe that such psychophysical experiments are not alternatives to phenomenological description; rather, they are forms of phenomenological description that are (more than usually) systematic and properly controlled. One benefit of relying on phenomenological descriptions that are systematic and that involve more than reports from a single subject (indeed, a subject who might be thought to be prejudiced by a philosophical agenda) is that they are much less open to flat denial by interlocutors who claim to introspect differently.

${ }^{2} \mathrm{I}^{\prime} 1 \mathrm{l}$ assume in what follows that perception has the kind of representational content that would legitimate this way of talking (for defense, see Byrne (2010); Pautz (2010); Siegel (2010); for criticism, see Travis (2004)). But I won't assume that such contents occur at the personal level. Given this last point, it is not a constraint on the attribution of a content to a perceptual state that the subject in whom the state occurs has conscious awareness of this content.

${ }^{3}$ This example figures centrally in classic 20th century philosophical writing about perception; for example, see Broad (1923, 235), Price (1964), Russell (1912).

${ }^{4} \mathrm{My}$ distinction between the constancy and inconstancy reactions echoes a contrast drawn by some writers between our reactions to objects' "objective"/"projective"/"distal" features, on the one hand, and to their "subjective" / "apparent" / "proximal" features, on the other. Unfortunately, it's often not so clear just what the contrast between objective and subjective (etc.) come to in these discussions, or why one set of reactions counts as more or less objective/projective/distal (etc.) than the others. I introduce new terminology in the hope that it is is less freighted with controversial theoretical assumptions than the traditional candidates (I'll return to this topic in §2).

${ }^{5}$ Tye and Byrne, in the works cited above, accept this view as a way of defending the representationalist/intentionalist thesis that (roughly) perceptual phenomenology supervenes on the representational content of perception. This thesis is threatened by puzzle cases since, on the one hand, there seems to be a phenomenological difference between, e.g., the perception of a tilted penny and the perception of a penny seen straight on, while on the other hand it might seem that in both cases there is identical representational content ascribing a certain shape to a particular penny. The dual property proposal is potentially helpful in defending the supervenience thesis because it supplies material from which we might find a difference between the representational 
contents of the two perceptual episodes to go with the phenomenal difference. However, in endorsing the dual property proposal, I don't need or intend to take sides on the supervenience thesis.

I should also note that, despite adverting to representational contents of states of the visual system, the dual property proposal is intended to be compatible with (at least many versions of) disjunctivism about perception (Hinton, 1973; McDowell, 1982). While disjunctivists deny that the relation between subjects and objects that is constitutive of veridical perception involves epistemic intermediation by representational contents, they do not deny that this relation involves causally intermediate states in the subpersonal visual system (inter alia), and there is nothing to prevent them from accepting that such subpersonal causally intermediate states have representational contents. The current proposal is in conflict with claims to the effect that subpersonal states of the visual system lack representational contents (e.g., Gibson, 1979; Brooks, 1991; O'Regan and Noë, 2002); but I take these claims to be highly implausible (Cohen, 2001), and so will set them aside.

${ }^{6}$ Below I'll deny that the second of these properties is best thought of as elliptical; but I'll stick by the current choice of labels for present purposes just to get the computational view on the table.

${ }^{7}$ Thus, I am proposing that the perceptual state of the subject is both (i) causally/computationally prior to the representation of an object feature such as round, and (ii) correlated with (hence, explanatory of) subjects' inconstancy reactions in the puzzle cases. However, in a pair of intriguing papers, Gilchrist (2010) and MacLeod (2010) argue against the idea (which they attribute to 20th century phenomenalists in philosophy and psychology) that there is any early perceptual stage that plays both of these roles. Rather, on their view, what plays role (ii) is causally posterior to the representation of object features.

While I regard the Gilchrist/MacLeod challenge as extremely important, I do not think it presents any insuperable obstacle for the position I am defending. One respect in which my view seems to me less vulnerable to their challenge than those they target directly is that the perceptual stage I am using to explain the inconstancy reactions is not itself subject to serious doubt. Unlike sense-data or the phenomenal given, as it might be, there is no question among the parties to the discussion that there is a gross state of the perceptual system at a time. Similarly, there is no serious reason to doubt that the gross state of the perceptual system at a time is causally prior to the representation by the perceptual system of object features. Given this background of agreement, my view makes the additional, non-trivial, broadly empirical, claim that that gross state of the perceptual system can also serve to explain subjects' inconstancy reactions. If I am correct in so claiming, then there is no reason to reject the present view. 
${ }^{8}$ This last qualification is important. As Jameson and Hurvich (1989) observe eloquently, there are in ecologically realistic settings a wide range of perceptually relevant features of the perceptual perspective. Hence there are typically far too many unknowns for the perceptual system to compute closed form solutions for the assignment of values to object features. Consequently, in order to compute the object features it needs, the perceptual system must rely default value assumptions about at least many perspectival features.

${ }^{9}$ There are literally thousands of experimental results supporting this general claim; readers are invited to consult some of the works already cited above, as well as the overviews of (some of) the empirical literatures provided by Jameson and Hurvich (1989); Walsh and Kulikowski (1998); Wagner (2006).

${ }^{10}$ Terminological caution: Some writers, such as Noë (2004), use 'perspectival feature' not for features of the perceptual perspective (as on my usage), but for something closer to what I am calling the perceptual state dispositions of objects.

${ }^{11}$ Thinking about the total perceptual state as a joint product of both object features and perspectival features is connected with (at least) two other traditions worth noting.

First, this framework recalls the Kantian understanding of empirical cognition as resulting from both the contribution made by the extra-mental world and also the contribution made by our cognitive and perceptual apparatus (the forms of intuition). An important difference, however, is that where, for Kant, the extra-worldly contribution to perception/cognition comes from the nature of our perceptual and cognitive faculties themselves, the extra-worldly factor I am pointing to is rather the ways in we are situated with respect to the objects of perception.

My notion of the total perceptual state is also related to what psychologists sometimes call the perceptual signal - understood as something like the total energy arriving at the sensory transducers at a time. While there are interesting relationships here, this is also not what I mean in talking about the perceptual state. For, crucially, I want to be able to talk about cases of interaction involving contributions causally downstream of transduction - cases where the interaction occurs not at the transduction stage, but at some later point. For example, there are many cases where our perceptual responses are shaped both by the energy striking our receptors (which arrives from outside the head of the subject) and by the state of adaptation of receptoral and postreceptoral systems (which are inside the head of the subject). When I talk about our total perceptual state, therefore, I mean a state of a total perceptual system, which is a result of current transduction of the gross perceptual signal, perceptual processing, and past states of the system. 
${ }^{12}$ This suggests an important further (empirical) question (to which, alas, I do not know the answer). Namely, given that the perceptual system can compute from perceptual state dispositions to either object feature or perspectival features, why does it carry out one of these computations rather than another on any given occasion? That is, what are the conditions under which the perceptual system will compute object features, and what are the conditions under which it will compute perspectival features? This seems to me a topic crying out for empirical study.

${ }^{13}$ It should also be noted that there is some evidence to the effect that perceptual representations of (at least some) perspectival features are less accurate/reliable than perceptual representations of object features or peceptual state dispositions (Rutherford and Brainard, 2002; Ripamonti et al., 2004; de Almeida and Nascimento, 2009). I take this not to impugn my claim that such features are, in fact, represented by perceptual systems.

${ }^{14}$ One consideration that might be thought to suggest that such computations are not always carried out is that, while subjects can be made to switch between responding to perceptual state dispositions (inconstancy reactions) and responding to object features (constancy reactions), there is no evidence of wavering, confusing, or averaging, in subject responses as one might expect were both properties always represented and available for report. The difficulty with this line of thought is that, while there is no evidence of wavering, confusing, or averaging in the responses tracking perceptual state dispositions (which I'm claiming can be represented in the absence of representation of object features), there is also no such evidence in the responses tracking object features (which, if I'm right, should only be represented when perceptual state dispositions are represented as well, since they are the computational input on the basis of which object features are derived). So I don't think this line of argument can establish the point.

${ }^{15}$ I should add that there are related cases that plausibly do involve cognitive inference working together with perceptual computation. For example, perceptual computation and cognitive inference might interact if the former assigns an object feature - say, a size - to my coffee cup that is at odds with my memory representation of the cup's size, or that is at odds with my general beliefs about the size of coffee cups. In such cases we might use cognition/inference as a way of constraining the interpretation of the perceptual state. We might conclude, say, that there is some otherwise unexpected effect of a perspectival feature (something funny must be going on with the light, or someone slipped something in my coffee). Or else we might revise other beliefs - perhaps concluding that the perceived object is not my coffee cup but a replacement, or that it is not really a coffee cup but a coffeecup-shaped and not-coffee-cup-sized sculpture. The contrast between these cases where cognitive inference is involved and the ordinary puzzle cases once 
again reinforces the conclusion that our computations should be distinguished from cognitive inference.

${ }^{16}$ I am grateful to Sarah Allred, Craig Callender, Don MacLeod, Mohan Matthen, Susanna Schellenberg, Susan Schneider, and an audience at the Workshop on Cognitive and Developmental Factors in Perceptual Constancy at the University of Pennsylvania for helpful discussion of the topics of this paper.

\section{References}

de Almeida, V. M. N. and Nascimento, S. M. C. (2009). Perception of illuminant colour changes across real scenes. Perception, 38(8), 1109-1117.

Arend, L. (1993). How much does illuminant color affect unattributed colors? Journal of the Optical Society of America A, 10, 2134-2147.

Arend, L. and Reeves, A. (1986). Simultaneous color constancy. Journal of the Optical Society of America A, 3(10), 1743-1751.

Arend, L., Reeves, A., Schirillo, J., and Goldstein, R. (1991). Simultaneous color constancy: patterns with diverse Munsell values. Journal of the Optical Society of America A, 8, 661-672.

Barbet, I. and Fagot, J. (2002). Perception of the corridor illusion by baboons. Behavioural Brain Research, 132(1), 111-115.

Bäuml, K.-H. (1999). Simultaneous colour constancy: how surface color perception varies with the illuminant. Vision Research, 39(8), 1531-1550.

Blackwell, K. T. and Buchsbaum, G. (1988). Quantitative studies in color constancy. Journal of the Optical Society of America A, 5, 1772-1780.

Broad, C. D. (1923). Scientific Thought. Harcourt, Brace and Company, New York.

Brooks, R. (1991). Intelligence without representation. Artificial Intelligence, 47, 139-159.

Byrne, A. (2002). Intentionalism defended. The Philosophical Review, 110, 199240.

Byrne, A. (2010). Experience and content. Philosophical Quarterly.

Byrne, A. and Hilbert, D. R., editors (1997). Readings on Color, Volume 2: The Science of Color. MIT Press, Cambridge, Massachusetts.

Chisholm, R. (1957). Perceiving: A Philosophical Study. Cornell University Press, Ithaca. 
Cohen, J. (2001). Whither visual representations? Whither qualia?: Open peer commentary on O'Regan and Noë (2002). Behavioral and Brain Sciences, 24(5), 980-981.

Cohen, L., Gray, F., Meyrignac, C., Dehaene, S., and Degos, J.-D. (1994). Selective deficit of visual size perception: two cases of hemimicropsia. Journal of Neurology, Neurosurgery, and Psychiatry, 57, 73-78.

Cornelissen, F. W. and Brenner, E. (1995). Simultaneous colour constancy revisited: an analysis of viewing strategies. Vision Research, 35, 2431-2448.

Dannemiller, J. L. (1989). A test of color constancy in 9- and 20-week-old human infants following simulated illuminant changes. Developmental Psychology, $25,171-184$.

Dannemiller, J. L. (1993). Rank orderings of photoreceptor photon catches from natural objects are nearly illuminant-invariant. Vision Research, 33, 131-140.

Dannemiller, J. L. and Hanko, S. A. (1987). A test of color constancy in 4-monthold human infants. Journal of Experimental Child Psychology, 44, 255-267.

Douglas, R. H., Eva, J., and Guttridge, N. (1988). Size constancy in goldfish (carassius auratus). Behavioural Brain Research, 30, 37-42.

Ducasse, C. J. (1942). Moore's refutation of idealism. In P. A. Schilpp, editor, The Philosophy of G. E. Moore, pages 225-252. Open Court Press, LaSalle, Illinois.

Ekroll, V., Faul, F., Niederée, R., and Richter, E. (2002). The natural center of chromaticity space is not always achromatic: A new look at colour induction. Proceedings of the National Academy of Sciences, 99(20), 13352-13356.

Fodor, J. A. (1983). The Modularity of Mind. MIT Press, Cambridge, Massachusetts.

Foster, D. H. and Nascimento, S. M. C. (1994). Relational colour constancy from invariant cone-excitation ratios. Proceedings of the Royal Society of London B, $257,115-121$.

Fujita, K. (1997). Perception of the Ponzo illusion by rhesus monkeys, chimpanzees and humans: similarity and difference in the three primate species. Perception \& Psychophysics, 59, 284-292.

Gibson, J. J. (1979). The Ecological Approach to Visual Perception. Houghton Mifflin, Boston.

Gilchrist, A. L. (1988). Lightness contrast and failures of constancy: A common explanation. Perception \& Psychophysics, 43, 415-424.

Gilchrist, A. L. (2010). Objective and subjective sides of perception. In S. Allred and G. Hatfield, editors, Visual Experience: Sensation, Cognition and Constancy. Oxford University Press, New York. 
Granrud, C. E. (2006). Size constancy in infants: 4-month-olds' responses to physical versus retinal image size. Experimental Psychology: Human Perception and Performance, 32(6), 1398-1404.

von Helmholtz, H. (1962). Helmholtz's Treatise on Physiological Optics. Dover, New York. Originally published in 1867.

Hinton, J. M. (1973). Experiences. Oxford University Press, Oxford.

Hurlbert, A. C. (1989). Cues to the color of the illuminant. Ph.D. thesis, Massachusetts Institute of Technology, Cambridge, Massachusetts.

Ingle, D. (1998). Perceptual constancies in lower vertebrates. In V. Walsh and J. J. Kulikowski, editors, Perceptual Constancy: Why Things Look as They Do, pages 173-191. Cambridge University Press.

Jameson, D. and Hurvich, L. M. (1989). Essay concerning color constancy. Annual Review of Psychology, 40, 1-22. Reprinted in Byrne and Hilbert (1997), 177-198.

Katz, D. (1935). The world of Color. Kegan, Paul, Trench Truber \& Co., London. translated by R. B. MacLeod and C. W. Fox.

Kelly, S. D. (2004). Seeing things in Merleau-Ponty. In T. Carman and M. B. N. Hansen, editors, The Cambridge Companion to Merleau-Ponty. Cambridge University Press, Cambridge.

MacLeod, D. I. A. (2010). A mechanistic perspective on the given. In S. Allred and G. Hatfield, editors, Visual Experience: Sensation, Cognition and Constancy. Oxford University Press, New York.

Mausfeld, R. (1998). Color perception: From Grassman codes to a dual code for object and illumination colors. In W. G. K. Backhaus, R. Kliegl, and J. S. Werner, editors, Color Vision: Perspectives from Different Disciplines, pages 219250. Walter de Gruyter, Berlin.

Mausfeld, R. (2003). The dual coding of colour: 'surface colour' and 'illumination colour' as constituents of the representational format of perceptual primitives. In R. Mausfeld and D. Heyer, editors, Colour Perception: Mind and the Physical World, pages 381-430. Oxford University Press, New York.

McDowell, J. (1982). Criteria, defeasibility and knowledge. Proceedings of the British Academy, 68, 455-479.

Neumeyer, C. (1998). Comparative aspects of color constancy. In V. Walsh and J. Kulikowski, editors, Perceptual Constancy: Why Things Look as They Do, pages 323-351. Cambridge University Press, Cambridge.

Noë, A. (2004). Action in Perception. MIT Press, Cambridge, Massachusetts. 
Noë, A. (2007). Real presence. Philosophical Topics, 33(1), 235-264.

O'Regan, J. K. and Noë, A. (2002). A sensorimotor account of vision and visual consciousness. Behavioral and Brain Sciences, 24(5), 883-975.

Pautz, A. (2010). An argument for the intentional view of visual experience. In B. Nanay, editor, Perceiving the World. Oxford University Press, New York.

Price, H. H. (1964). Appearing and appearances. American Philosophical Quarterly, 1, 3-19.

Ripamonti, C., Bloj, M., Hauck, R., Kiran, M., Greenwald, S., Maloney, S. I., and Brainard, D. H. (2004). Measurements of the effect of surface slant on perceived lightness. Journal of Vision, 4(9), 747-63.

Russell, B. (1912). The Problems of Philosophy. Oxford University Press, London.

Rutherford, M. D. and Brainard, D. H. (2002). Lightness constancy: a direct test of the illumination-estimation hypothesis. Psychological Science, 13(2), 142-149.

Rüttiger, L., Braun, D. I., Gegenfurtner, K. R., Petersen, D., Schönle, P., and Sharpe, L. T. (1999). Selective color constancy deficits after circumscribed unilateral brain lesions. The Journal of Neuroscience, 19(8), 3094-3106.

Schellenberg, S. (2008). The situation-dependency of perception. The Journal of Philosophy, 105(2), 55-84.

Sellars, W. (1963). Phenomenalism. In W. Sellars, editor, Science, Perception and Reality. Routledge \& Kegan Paul, London.

Sellars, W. (1975). The adverbial theory of the objects of perception. Metaphilosophy, 6, 144-160.

Shevell, S. K. (1978). The dual role of chromatic backgrounds in color perception. Vision Research, 18, 1649-1661.

Siegel, S. (2010). The weak content view. In B. Nanay, editor, Perceiving the World. Oxford University Press, New York.

Slater, A., Mattok, A., and Brown, E. (1990). Size constancy at birth: Newborn infants' responses to retinal and real size. Journal of Experimental Child Psychology, 49, 314-322.

Travis, C. (2004). The silence of the senses. Mind, 113(449), 57-94.

Troost, J. M. and deWeert, C. M. M. (1991). Naming versus matching in color constancy. Perception E Psychophysics, 50, 591-602.

Tye, M. (1996). Perceptual experience is a many layered thing. Philosophical Issues, 7, 117-126. 
Tye, M. (2002). Visual qualia and visual content revisited. In D. J. Chalmers, editor, Philosophy of Mind: Classical and Contemporary Readings, pages 447456. Oxford University Press, New York.

Valberg, A. and Lange-Malecki, B. (1990). 'Colour constancy' in Mondrian patterns: A partial cancellation of physical chromaticity shifts by simultaneous contrast. Vision Research, 30(3), 371-380.

Wagner, M. (2006). The Geometries of Visual Space. Lawrence Earlbaum, Mahwah, N.J.

Walraven, J. (1976). Discounting the background - the missing link in the explanation of chromatic induction. Vision Research, 16, 289-295.

Walsh, V. and Kulikowski, J., editors (1998). Perceptual Constancy: Why Things Look As They Do. Cambridge University Press, Cambridge.

Zaidi, Q. (1998). Identification of illuminany and object colors: Heuristic-based algorithms. Journal of the Optical Society of America A, 15, 1767-1776.

Zaidi, Q. (2001). Color constancy in a rough world. Color Research and Application, 26, S192-S200. 


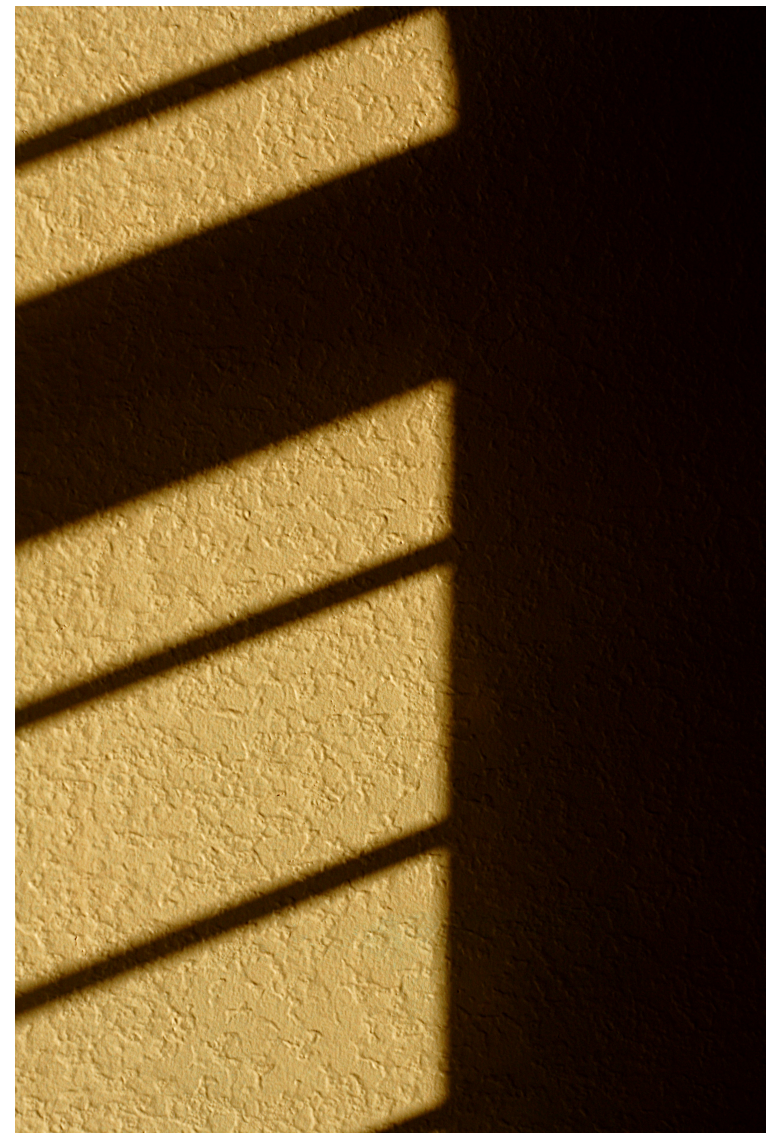

Figure 1: Non-uniformly illuminated but uniformly painted wall. Photograph (C) Jonathan Cohen. 


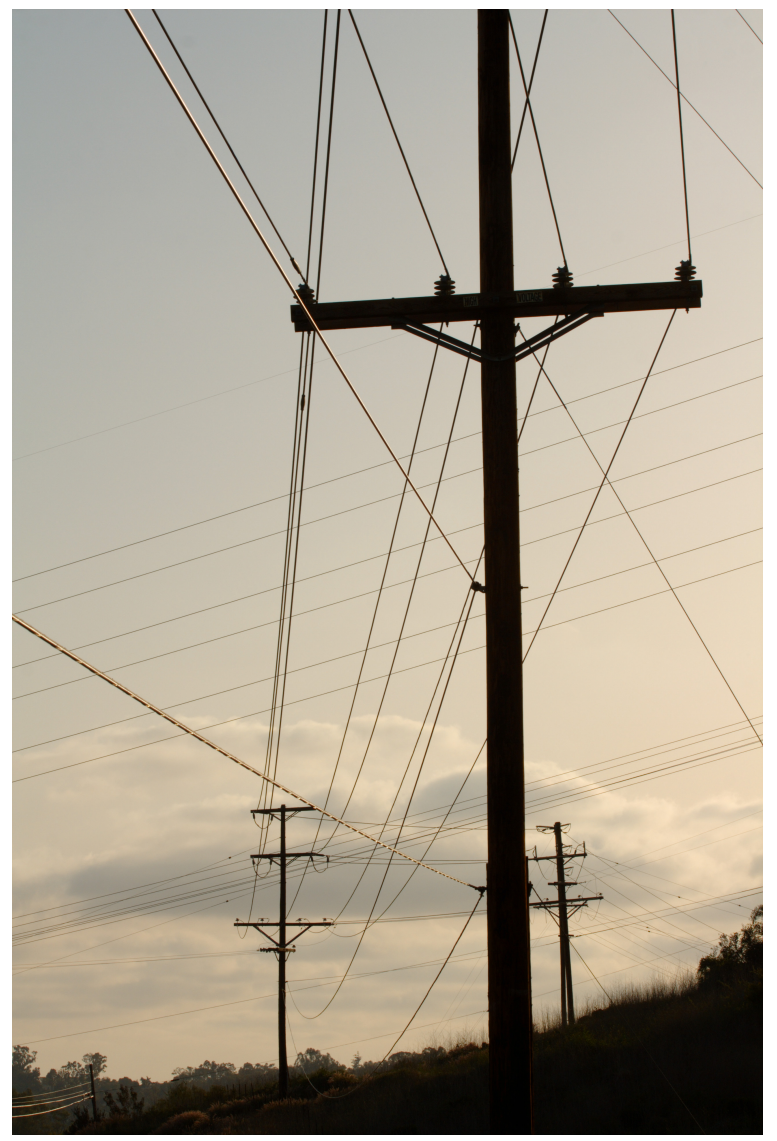

Figure 2: Telephone poles receding into the distance. Photograph (C) Jonathan Cohen.

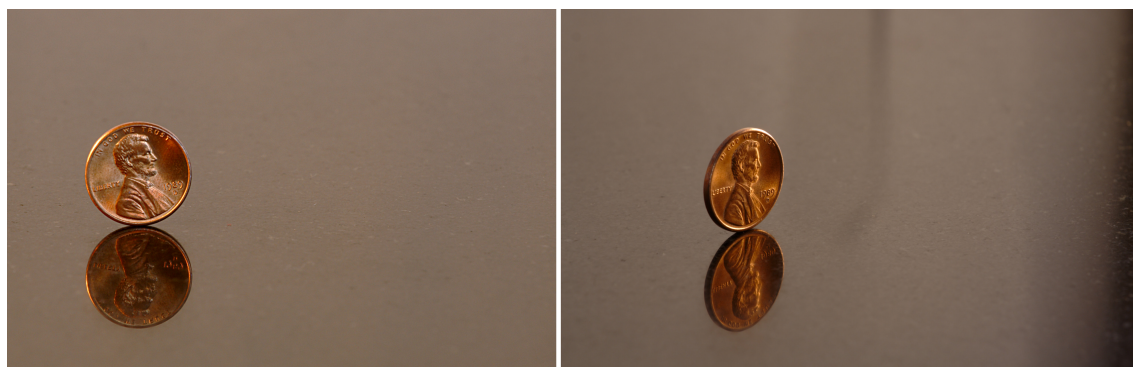

Figure 3: Penny as seen from two angles. Photograph (C) Jonathan Cohen. 